\title{
Prevision Domains and Convex Powercones ${ }^{\star}$
}

\author{
Jean Goubault-Larrecq \\ LSV, ENS Cachan, CNRS, INRIA Futurs \\ 61, av. du président-Wilson, 94230 Cachan, France \\ goubaultelsv.ens-cachan.fr
}

\begin{abstract}
Two recent semantic families of models for mixed probabilistic and non-deterministic choice over a space $X$ are the convex powercone models, due independently to Mislove, and to Tix, Keimel, and Plotkin, and the continuous prevision model of the author. We show that, up to some minor details, these models are isomorphic whenever $X$ is a continuous, coherent cpo, and whether the particular brand of non-determinism we focus on is demonic, angelic, or chaotic. The construction also exhibits domains of continuous previsions as retracts of well-known continuous cpos, providing simple bases for the various continuous cpos of continuous previsions. This has practical relevance to computing approximations of operations on previsions.
\end{abstract}

\section{Introduction}

Continuous lower and upper previsions, and forks, were proposed in [5] as adequate models for mixed non-deterministic (demonic, angelic, and chaotic respectively) and probabilistic choice. At the end of this paper, it was claimed that there was a strong relation between this model and that discovered independently by Mislove [13] and by Tix [16 17], consisting of convex non-empty subsets of continuous valuations, which are also compact saturated, resp. closed, resp. (compact) lenses-the so-called convex powercones. We make this connection more precise, and to show that this "strong relation" is in fact an isomorphism, provided $X$ is a coherent continuous pointed cpo.

Before we go on, let us mention that Keimel and Plotkin [10] solved a very similar problem, under the guise of finding predicate transformers characterizing convex powercones. Keimel and Plotkin's so-called functional representations of predicate transformers map elements of convex powercones over a cpo $X$ to certain continuous functionals very much like our continuous previsions (essentially, up to the replacement of $\mathbb{R}^{+}$by $\overline{\mathbb{R}^{+}}=\mathbb{R}^{+} \cup\{+\infty\}$ ). However, Keimel and Plotkin's convex powercones are composed of convex subsets of continuous valuations, and the latter may be unbounded. In practice, convex subsets of continuous probabilities (such that the measure of the whole space is 1) or subprobabilities (at most 1) seem to fit more tightly our needs in modeling probabilistic choice, and Keimel and Plotkin note that "it would be more natural, from the point of view of computer science applications, to restrict to subprobability valuations, rather than allowing all of them." This is what we do here.

Our isomorphism result is not a consequence of the theorems of Keimel and Plotkin, although it is likely that adapting their proofs (and in fact, making them murkier) would

\footnotetext{
^ Partially supported by the INRIA ARC ProNoBis.
}

R. Amadio (Ed.): FOSSACS 2008, LNCS 4962, pp. 318 333. 2008.

(C) Springer-Verlag Berlin Heidelberg 2008 
give us the desired results. We take the route of [7, Section 11.7], and prove the isomorphism in two steps: first, isomorphism theorems on fairly general classes of topological spaces $X$, but where convexity has to be replaced by a slightly stronger notion; second, the proof that the strong notions of convexity coincide with ordinary convexity on coherent continuous pointed cpos.

Outline. We quickly go over preliminaries in Section 2 , then show our first isomorphism theorem in the demonic case, in Section 3. This relies on the notion of strong convexity, and uses notions of barycenters à la Choquet-Bishop-de Leeuw. Showing that strong convexity reduces to convexity in the case of cpos is the subject of Section 4 and is the only section that relies on theorems by Tix, Keimel, and Plotkin. We deal with the angelic case in Section 5, which we reduce to the demonic case by the socalled convex-concave duality. The chaotic case is now ripe for treatment in Section 6 We conclude in Section[7

Related Work. Clearly [16]17|10] and [5] are most relevant. Other relevant material will be cited on the fly.

\section{Preliminaries}

See [1]3|12] for background material on domain theory and topology. A cpo $X$ is a partially ordered set (poset) in which every directed set has a least upper bound, or sup. We write $\leq$ its ordering. The Scott topology on a poset has as opens all upward-closed subsets $U$ such that whenever $\left(z_{i}\right)_{i \in I}$ is a directed family having a sup $z$ in $U$, then some $z_{i}$ is in $U$ already. The way-below relation $\ll$ on a poset is defined by $x \ll y$ iff whenever $\left(z_{i}\right)_{i \in I}$ is a directed family having a $\sup z$ with $y \leq z$, then $x \leq z_{i}$ for some $i \in I$. A poset $X$ is continuous iff every $x \in X$ is the directed sup of all elements $y \ll x$. A basis of $X$ is then a subset $B$ of $X$ such that every $x \in X$ is the directed sup of all elements $y \in B$ such that $y \ll x$. The Scott topology then has a basis of open sets of the form $\uparrow y=\{x \in X \mid y \ll x\}, y \in B$.

For any topological space (e.g., cpo) $X$, let $\left\langle X \rightarrow \mathbb{R}^{+}\right\rangle$be the poset of all bounded continuous maps from $X$ to $\mathbb{R}^{+}$. We take $\mathbb{R}^{+}$with the Scott topology, whose non-trivial opens are the open intervals $(t,+\infty), t \in \mathbb{R}^{+}$, and order $\left\langle X \rightarrow \mathbb{R}^{+}\right\rangle$pointwise. A continuous prevision $F$ on a topological space (e.g., a cpo) $X$ [5] is a Scott-continuous map from $\left\langle X \rightarrow \mathbb{R}^{+}\right\rangle$to $\mathbb{R}^{+}$such that $F(a f)=a F(f)$ for every $a \in \mathbb{R}^{+}$(positive homogeneity). A prevision $F$ is lower iff $F\left(h+h^{\prime}\right) \geq F(h)+F\left(h^{\prime}\right)$ for every $h, h^{\prime}$, upper iff $F\left(h+h^{\prime}\right) \leq F(h)+F\left(h^{\prime}\right)$ for every $h, h^{\prime}$, linear iff $F\left(h+h^{\prime}\right)=F(h)+$ $F\left(h^{\prime}\right)$, normalized iff $F(a+h)=a+F(h)$ for every function $h$ and constant $a \in \mathbb{R}^{+}$, subnormalized iff $F(a+h) \leq a+F(h)$ for every $h$ and constant $a$. A fork is a pair $\left(F^{-}, F^{+}\right)$of continuous previsions, where $F^{-}$is lower, $F^{+}$is upper, and Walley's condition $F^{-}\left(h+h^{\prime}\right) \leq F^{-}(h)+F^{+}\left(h^{\prime}\right) \leq F^{+}\left(h+h^{\prime}\right)$ holds for every $h, h^{\prime}$. A fork is normalized, resp. sub-normalized, whenever both $F^{-}$and $F^{+}$are. We shall concentrate on normalized previsions and forks in the sequel.

It was shown in [5] that, among continuous normalized previsions, the lower brand was an adequate model of mixed probabilistic and demonically non-deterministic choice, the upper brand was one of mixed probabilistic and angelically non-deterministic 
choice, while normalized forks were an adequate model of mixed probabilistic and chaotically non-deterministic choice. It was essentially well-known since Tix [15] that the space of continuous (subnormalized, resp. normalized) linear previsions were isomorphic to Jones' space $\mathbf{V}_{\leq 1}(X)$ (resp., $\mathbf{V}_{1}(X)$ ) of subprobability (resp. probability) valuations. A valuation is a map $p$ from the set $\mathcal{O}(X)$ of all opens of $X$ to $\overline{\mathbb{R}^{+}}$ that is strict $(p(\emptyset)=0)$, monotone $(U \subseteq V$ implies $p(U) \leq p(V))$, and modular $(p(U \cup V)+p(U \cap V)=p(U)+p(V))$. Subprobability valuations (resp., probability valuations) are those such that $p(X) \leq 1$ (resp., $p(X)=1$ ). The valuation $p$ is continuous iff $p\left(\bigcup_{i \in I} U_{i}\right)=\sup _{i \in I} p\left(U_{i}\right)$ for every directed family $\left(U_{i}\right)_{i \in I}$ of opens of $X$. We shall always equip each cpo, and in fact each poset, $X$ with its Scott topology. However, we shall consider more general topological spaces in the sequel. For every open $U$, let $\chi_{U}$ map $x$ to 1 if $x \in U$, to 0 otherwise. The isomorphism between the space $\mathbf{P}_{1}^{\triangle}(X)$ of continuous normalized linear previsions $G$ on $X$ and $\mathbf{V}_{1}(X)$ maps $G$ to $p=\gamma_{\mathcal{e}}(G)$ defined by $p(U)=G\left(\chi_{U}\right)$ for every open $U$, and conversely, maps $p$ to $G=\alpha_{\mathcal{C}}(p)$ defined by letting $G(h)$ be the Choquet integral of $h$ along $p$ [5]. (The Choquet integral of $h \in\left\langle X \rightarrow \mathbb{R}^{+}\right\rangle$along $p$, which we shall write $\oint_{x \in X} h(x) d p$, is defined as the ordinary Riemann integral $\int_{0}^{+\infty} p\left(h^{-1}(t,+\infty)\right) d t$. This is a continuous linear prevision of $h$ and is also linear and Scott-continuous in $p$, whenever $p$ is a continuous valuation. Note that Choquet integration is also defined when $p$ is merely a so-called game [4].)

Let $\nabla \mathbf{P}_{1}(X)$ be the space of continuous normalized lower previsions on $X, \mathbf{P}_{1}^{\triangle}(X)$ that of all continuous normalized linear previsions (with the Scott topology, ordered pointwise), and $\mathbf{P}_{1 \text { wk }}^{\triangle}(X)$ the same space with the weak topology, defined as the smallest that contains the subbasic opens $[f>r]=\left\{G \in \mathbf{P}_{1 w k}^{\triangle}(X) \mid G(f)>r\right\}, f \in$ $\left\langle X \rightarrow \mathbb{R}^{+}\right\rangle, r \in \mathbb{R}^{+}$. The Scott topology is always finer than the weak topology. When $X$ is a continuous cpo with a least element, both topologies coincide, i.e., $\mathbf{P}_{1 w k}^{\triangle}(X)=$ $\mathbf{P}_{1}^{\triangle}(X)$. This is easily obtained from the coincidence of the two topologies on spaces of valuations, through the isomorphism between continuous valuations and continuous linear previsions above (see [9], who refers to Tix [15, Satz 4.10], who cites Kirch [11, Satz 8.6]; see also [7, Proposition 3.7.12].)

The relation between spaces of previsions and sets of convex subsets of valuations alluded to in the introduction takes the following form, in the demonic case [5], Proposition 4]. Let the Smyth powerdomain $Q(Y)$ of a topological space be the set of all non-empty compact saturated subsets (see below) of $Y$, ordered by reverse inclusion $\supseteq$. (This is a standard model of demonic non-determinism alone [1].) Then, there is a map CCoeur $_{1}: \nabla \mathbf{P}_{1}(X) \rightarrow \mathcal{Q}\left(\mathbf{P}_{1 w k}^{\triangle}(X)\right)$ sending each continuous normalized lower prevision $F$ to its heart $C$ Coeur $1(F)=\left\{G \in \mathbf{P}_{1}^{\triangle}(X) \mid F \leq G\right\}$. Whenever $X$ is stably compact (see below), the heart is non-empty and compact saturated. (Both properties are non trivial.) Moreover, the heart is convex: for any two $G, G^{\prime} \in C C_{\text {Coeur }}(F)$, for any $\alpha \in[0,1], \alpha G+(1-\alpha) G^{\prime}$ is in $\operatorname{CCoeur}_{1}(F)$ (trivial). Conversely, there is a map $\sqcap: \mathcal{Q}\left(\mathbf{P}_{1 w k}^{\triangle}(X)\right) \rightarrow \nabla \mathbf{P}_{1}(X)$, sending $\mathcal{Q}$ to $\lambda h \in\left\langle X \rightarrow \mathbb{R}^{+}\right\rangle \cdot \min _{G \in \mathcal{Q}} G(h)$. (The min is, indeed, attained.) CCoeur ${ }_{1}$ and $\Pi$ are Scott-continuous, and form a Galois insertion, i.e., $П \circ C$ Coeur $_{1}=$ id (Rosenmuller's Theorem), and $C$ Coeur $_{1} \circ \sqcap \supseteq$ id. We shall denote Galois connections, i.e., pairs of monotone maps $\alpha, \gamma$ such that $\alpha \circ \gamma \leq$ id and id $\leq \gamma \circ \alpha$, by $\alpha \dashv \gamma$; so CCoeur $r_{1} \dashv \sqcap$ (recall that $\leq$ is $\supseteq$ on $\left.Q\left(\mathbf{P}_{1 w k}^{\triangle}(X)\right)\right)$. 
A subset $Q$ of $X$ is compact iff one can extract a finite subcover from every open cover of $Q$. It is saturated iff it is the intersection of all opens containing it, a.k.a. it is upward-closed in the specialization quasi-ordering $\leq$, defined by $x \leq y$ iff every open containing $x$ contains $y$. A topological space $X$ is stably compact (taking Jung's definitions [9]) iff $X$ is $T_{0}$ ( $\leq$ is an ordering), well-filtered (for every filtered family $\left(Q_{i}\right)_{i \in I}$ of compact saturated subsets, for every open $U$, if $\bigcap_{i \in I} Q_{i} \subseteq U$ then $Q_{i} \subseteq U$ already for some $i \in I$ ), locally compact (whenever $x \in U$ with $U$ open, there is a compact saturated subset $Q$ such that $x \in \operatorname{int}(Q) \subseteq Q \subseteq U$, where $\operatorname{int}(Q)$ denotes the interior of $Q$ ), coherent (the intersection of any two compact saturated subsets is again so) and compact. Every continuous cpo $X$ is well-filtered and locally compact. If additionally $X$ is pointed, i.e., has a least element, then $X$ is compact. If finally $X$ is also coherent, then $X$ is stably compact. Stable compactness has a long history, going back to Nachbin (1948; see [9]).

\section{Demonic Non-determinism + Probabilistic Choice}

The central question of this paper, in the demonic case, is whether the pair CCoeur $_{1} \dashv$ $\Pi$ actually defines an isomorphism between $\nabla \mathbf{P}_{1}(X)$ and some suitable subset of $\mathcal{Q}\left(\mathbf{P}_{1 w k}^{\triangle}(X)\right)$. One natural candidate is $\mathcal{Q}^{c v x}\left(\mathbf{P}_{1 w k}^{\triangle}(X)\right)$, the space of all elements of $\mathcal{Q}\left(\mathbf{P}_{1 w k}^{\triangle}(X)\right)$ that are convex-since the heart is always convex.

However, the right notion we need is that of strong convexity, defined below. (Connoisseurs will note that the same idea is the root of the classic Choquet-Bishop-de Leeuw extension to the Krein-Milman Theorem.) One first notes that convex sets 2 of linear previsions are those that are stable by taking finite barycenters, i.e., such that for any finite set $G_{0}, G_{1}, \ldots, G_{n}$ of $n+1$ elements of 2 , for any coefficients $a_{0}, a_{1}, \ldots, a_{n} \in \mathbb{R}^{+}$with $\sum_{i=0}^{n} a_{i}=1, \sum_{i=0}^{n} a_{i} G_{i}$ is again in 2 . On any space $Y$, the Dirac valuation $\delta_{y}$ defined so that $\delta_{y}(V)=1$ if $y \in V, 0$ otherwise, is a continuous probability valuation, and so are the simple probability valuations of the form $\sum_{i=0}^{n} a_{i} \delta_{y_{i}}$, with $a_{0}, a_{1}, \ldots, a_{n}$ as above. The Choquet integral of $h \in\left\langle Y \rightarrow \mathbb{R}^{+}\right\rangle$ along such a simple probability valuation yields $\sum_{i=0}^{n} a_{i} h\left(y_{i}\right)$. It follows that we can rewrite the finite barycenter $\sum_{i=0}^{n} a_{i} G_{i}$ as $\lambda h \in\left\langle X \rightarrow \mathbb{R}^{+}\right\rangle \cdot \oint_{G \in \mathbf{P}_{1 \text { wk }}^{\triangle}(X)} G(h) d \mathcal{P}$, where $\mathcal{P}$ is the simple probability valuation $\sum_{i=0}^{n} a_{i} \delta_{G_{i}}$ on $\mathbf{P}_{1 w k}^{\triangle}(X)$ (an element of $\left.\mathbf{P}_{1}^{\triangle}\left(\mathbf{P}_{1 w k}^{\triangle}(X)\right) !\right)$. This allows us to define the general notion of barycenter Bary $(\mathcal{P})$ of a continuous probability valuation $\mathcal{P} \in \mathbf{P}_{1}^{\triangle}\left(\mathbf{P}_{1 w k}^{\triangle}(X)\right)$ as $\lambda h \in\left\langle X \rightarrow \mathbb{R}^{+}\right\rangle$. $\oint_{G \in \mathbf{P}_{1 w k}^{\triangle}(X)} G(h) d \mathcal{P} \in \mathbf{P}_{1}^{\triangle}(X)$.

Say that $\mathcal{P}$ is supported on $\mathcal{Q} \subseteq \mathbf{P}_{1 w k}^{\triangle}(X)$ iff $\mathcal{P}(\mathcal{U})=1$ for every open set of continuous probability valuations containing $\mathcal{Q}$. This intuitively says that $\mathcal{P}$ bears no mass outside $\mathcal{Q}$. One can check that $\sum_{i=0}^{n} a_{i} G_{i}$ is supported on $\mathcal{Q}$ (where $\mathcal{Q}$ is upwardclosed) iff $G_{i} \in \mathcal{Q}$ for all $i$ such that $a_{i} \neq 0$.

We then say that $\mathcal{Q}$ is strongly convex iff $\operatorname{Bary}(\mathcal{P}) \in \mathcal{Q}$ for every $\mathcal{P} \in \mathbf{P}_{1}^{\triangle}\left(\mathbf{P}_{1 w k}^{\triangle}(X)\right)$ that is supported on $\mathcal{Q}$. Whenever $\mathcal{Q}$ is strongly convex, this property must hold whenever $\mathcal{P}$ is a simple probability valuation, showing that every strongly convex upwardclosed set is convex. The converse fails, as we now show. Let $X$ be $\mathbb{N} \cup\{+\infty\}$, with the usual ordering and its Scott topology. Let $\mathcal{Q}$ be the set of all linear previsions of the 
form $\lambda h \cdot \sum_{i=0}^{n} a_{i} h\left(k_{i}\right)$, where $a_{0}, \ldots, a_{n}$ are as above and $k_{i} \in \mathbb{N}$. This is the convex hull of the set of linear previsions of the form $\alpha_{\mathrm{e}}\left(\delta_{k}\right), k \in \mathbb{N}$, and is therefore convex. (Recall that $\alpha_{\mathcal{e}}\left(\delta_{k}\right)$ is the image of $\delta_{k}$ through the isomorphism between continuous valuations and continuous linear previsions, and maps $h$ to $h(k)$.) It is clear that $\delta_{+\infty}$ is not in $\mathcal{Q}$. However, $\delta_{+\infty}$ arises as $\operatorname{Bary}\left(\delta_{\delta_{+\infty}}\right)$, and we check that $\delta_{\delta_{+\infty}}$ is supported on 2 : any open containing 2 must contain, say, $\delta_{0}$, hence also $\delta_{+\infty}$, since $\delta_{0} \leq \delta_{+\infty}$.

Proposition 1. Let $X$ be stably compact, and $F \in \nabla \mathbf{P}_{1}(X)$. Then $\operatorname{CCoeur}_{1}(F)$ is strongly convex.

Proof. We first observe that, given any compact saturated subset $Q$ of a space $Y$, given any continuous probability valuation $p$ on $Y$ that is supported on $Q$, for any $h \in\langle Y \rightarrow$ $\left.\mathbb{R}^{+}\right\rangle:(*) \oint_{y \in Y} h(y) d p \geq \min _{y \in Q} h(y)$. Let $a=\min _{y \in Q} h(y)$ (which is attained since $Q$ is compact). For all $t<a, f^{-1}(t,+\infty)$ contains $Q$, so $p\left(f^{-1}(t,+\infty)\right)=1$; hence $\oint_{y \in Y} h(y) d p=\int_{0}^{+\infty} p\left(f^{-1}(t,+\infty)\right) d t \geq \int_{0}^{a} p\left(f^{-1}(t,+\infty)\right) d t=a$.

For $f$ an arbitrary element of $\left\langle X \rightarrow \mathbb{R}^{+}\right\rangle$, apply $(*)$ to the case $Y=\mathbf{P}_{1 w k}^{\triangle}(X), p=$ $\mathcal{P}$ supported on $\mathcal{Q}=\operatorname{CCoeur}_{1}(F)$, taking $h(G)=G(f)$. (Note that $h$ is continuous, precisely because $Y$ is equipped with the weak topology.) We get $\left.\oint_{G \in \mathbf{P}_{1 w k}}(X)\right) G(f) d \mathcal{P}$ $\geq \min _{G \in \mathcal{Q}} G(f)=\prod \mathcal{Q}(f)$. However, remember that $\prod \circ C$ Coeur $r_{1}=$ id, so $\Pi \mathcal{Q}=$ $F$. Also, $\oint_{G \in \mathbf{P}_{1 w k}^{\triangle}(X)} G(f) d \mathcal{P}=\operatorname{Bary}(\mathcal{P})(f)$. So $\operatorname{Bary}(\mathcal{P}) \geq F$, i.e., $\operatorname{Bary}(\mathcal{P}) \in$ CCoeur $_{1}(F)$.

The converse direction, that strongly convex non-empty compact saturated subsets of $\mathbf{P}_{1 w k}^{\triangle}(X)$ arise from some element of $\nabla \mathbf{P}_{1}(X)$, relies on the following key Proposition 2. To appreciate it, look at the case when $\mathcal{Q}$ is the upward closure $\uparrow\left\{G_{1}, \ldots, G_{n}\right\}$ of $\left\{G_{1}, \ldots, G_{n}\right\}$ in $\mathbf{P}_{1 w k}^{\triangle}(X)$ : up to some details, the proposition states that if for each $h$, there is an $i$ with $G(h) \geq G_{i}(h)$ (not necessarily the same $i$ for each $h$ ), then there are coefficients $a_{0}, a_{1}, \ldots, a_{n}$ with $\sum_{i=0}^{n} a_{i}=1$ such that $G(h) \geq \sum_{i=0}^{n} a_{i} G_{i}(h)$ for all $h$. (This is similar to a key step in some proofs of the minimax theorem.)

The proof relies on Roth's Sandwich Theorem ([14], [17, Theorem 3.1]), which states that on every ordered cone $C$, for every positively homogeneous super-additive function $q: C \rightarrow \overline{\mathbb{R}^{+}}$and every positively homogeneous sub-additive function $p: C \rightarrow$ $\overline{\mathbb{R}^{+}}$such that $a \leq b$ implies $q(a) \leq p(b)$ (e.g., when $q \leq p$ and either $q$ or $p$ is monotonic), then there is a monotonic linear function $f: C \rightarrow \overline{\mathbb{R}^{+}}$such that $q \leq f \leq p$. A cone is a set $C$, together with a binary operation + turning it into a commutative monoid and a scalar multiplication $\cdot$ from $\mathbb{R}^{+} \times C$ to $C$, such that $1 \cdot a=a, 0 \cdot a=0$, $(r s) \cdot a=r \cdot(s \cdot a), r \cdot(a+b)=r \cdot a+r \cdot b$, and $(r+s) \cdot a=r \cdot a+s \cdot a$. An ordered cone is equipped in addition with a partial ordering $\leq$ making + and $\cdot$ monotonic. The function $q$ is positively homogeneous iff $q(s \cdot a)=s q(a)$ for all $s \in \mathbb{R}^{+}$, super-additive iff $q(a+b) \geq q(a)+q(b)$, sub-additive iff $q(a+b) \leq q(a)+q(b)$, and linear iff it has all three properties.

Proposition 2. Let $X$ be stably compact,, 2 be a non-empty compact saturated subset of $\mathbf{P}_{1 w k}^{\triangle}(X), G \in \mathbf{P}_{1 w k}^{\triangle}(X)$ such that $\Pi \mathcal{Q} \leq G$. Then there is a continuous probability valuation $\mathcal{P}$ on $\mathbf{P}_{1 \text { wk }}^{\triangle}(X)$ that is supported on $\mathcal{Q}$, and such that Bary $(\mathcal{P}) \leq G$. 
Proof. Consider the ordered cone $C=\left\langle\mathbf{P}_{1 w k}^{\triangle}(X) \rightarrow \mathbb{R}^{+}\right\rangle$, with the obvious + and $\cdot$, and the pointwise ordering. For every $\varphi \in\left\langle\mathbf{P}_{1 w k}^{\triangle}(X) \rightarrow \mathbb{R}^{+}\right\rangle$, let $q(\varphi)=$ $\min _{G \in \mathcal{Q}} \varphi(G)$. This is clearly positively homogeneous, super-additive, and monotonic. In fact, $q$ is even (Scott-)continuous: recall from [4] that, for any compact saturated subset $Q$ of a space $Y$, the unanimity game $\mathfrak{u}_{Q}$ (mapping each open $U$ containing $Q$ to 1 and all others to 0) is a continuous game, that the Choquet integral $\oint_{y \in Y} f(y) d \mathfrak{u}_{Q}$ equals $\min _{y \in Q} f(y)$ — so that $q(\varphi)=\oint_{G \in \mathbf{P}_{1 w k}^{\triangle}(X)} \varphi(G) d \mathfrak{u}_{\mathcal{Q}}$ —and that Choquet integration wrt. a continuous game is Scott-continuous in the integrated function.

Let $p(\varphi)=\inf \underset{\forall \in\left(x \in\left\langle X \rightarrow \mathbb{R}^{+}\right\rangle\right.}{\forall G^{\prime} \in \mathcal{Q} \cdot \varphi\left(G^{\prime}\right) \leq G^{\prime}(f)} G(f)$. (We take this to mean $+\infty$ if the inf is taken over an empty family of values.) It is easy to see that $p(a \varphi)=a p(\varphi)$ for every $a \in \mathbb{R}^{+}$: the case $a=0$ works by realizing that $f=0$ satisfies $\varphi\left(G^{\prime}\right)=G^{\prime}(f)$ for all $G \in \mathcal{Q}$, and then $G(f)=0$, the case $a \neq 0$ works by substituting $f / a$ for $f$ in the formula for $p$. Checking that $p$ is super-additive is only slightly harder: $p(\varphi)+p\left(\varphi^{\prime}\right)$ equals the inf of $G(f)+G(g)=G(f+g)$ when $f$ and $g$ range over functions such that $\varphi\left(G^{\prime}\right) \leq G^{\prime}(f)$ and $\varphi\left(G^{\prime}\right) \leq G^{\prime}(g)$ for all $G^{\prime} \in \mathbf{P}_{1 w k}^{\triangle}(X)$. Since every such $G^{\prime}$ is linear, they all satisfy $\left(\varphi+\varphi^{\prime}\right)\left(G^{\prime}\right) \leq G^{\prime}(f+g)$, so $p(\varphi)+p\left(\varphi^{\prime}\right)$ is greater than of equal to the inf of $G(f+g)$ when $f$ and $g$ satisfy the weaker condition $\left(\varphi+\varphi^{\prime}\right)\left(G^{\prime}\right) \leq G^{\prime}(f+g)$. This is then greater than or equal to $p\left(\varphi+\varphi^{\prime}\right)$.

We now check that $q \leq p$. This is where we use the assumption $\Pi \mathcal{Q} \leq G$. Fix $\varphi \in\left\langle\mathbf{P}_{1 w k}^{\triangle}(X) \rightarrow \mathbb{R}^{+}\right\rangle$. For all $f \in\left\langle X \rightarrow \mathbb{R}^{+}\right\rangle$such that $\forall G^{\prime} \in \mathcal{Q} \cdot \varphi\left(G^{\prime}\right) \leq G^{\prime}(f)$, we have $G(f) \geq \prod \mathcal{Q}(f)=\min _{G^{\prime} \in \mathcal{Q}} G^{\prime}(f) \geq \min _{G^{\prime} \in \mathcal{Q}} \varphi\left(G^{\prime}\right)=q(\varphi)$. Now take infs over $f$ on each side, whence $p(\varphi) \geq q(\varphi)$.

Using Roth's Sandwich Theorem, there is a monotonic linear functional $\mathcal{G}_{0}$ from $\left\langle\mathbf{P}_{1 w k}^{\triangle}(X) \rightarrow \mathbb{R}^{+}\right\rangle$to $\overline{\mathbb{R}^{+}}$such that $q \leq \mathcal{G}_{0} \leq p$. We claim that $\mathcal{G}_{0}$ never takes the value $+\infty$. Indeed, for any $\varphi \in\left\langle\mathbf{P}_{1 w k}^{\triangle}(X) \rightarrow \mathbb{R}^{+}\right\rangle$, letting $a=\sup _{G^{\prime} \in \mathbf{P}_{1 w k}}{ }_{1 X)} \varphi\left(G^{\prime}\right)$, $\mathcal{G}_{0}(\varphi) \leq p(\varphi) \leq a p\left(\chi_{\mathbf{P}_{1 w k}^{\triangle}(X)}\right) \geq G\left(\chi_{X}\right)=1$ because we may take $f=\chi_{X}$ in the definition of $p$, as $\varphi\left(G^{\prime}\right) \leq a=a G^{\prime}\left(\chi_{X}\right), G^{\prime}$ being normalized. So $\mathcal{G}_{0}$ is a linear prevision. Since $q \leq \mathcal{S}_{0}$ and $q\left(\chi_{\mathbf{P}_{1 w k}^{\triangle}(X)}\right)=1$, it follows that $\mathcal{G}_{0}\left(\chi_{\mathbf{P}_{1 w k}^{\triangle}(X)}\right)=1$; since $\mathcal{G}_{0}$ is linear, this is enough to show that $\mathcal{G}_{0}$ is normalized.

But $\mathcal{G}_{0}$ is not necessarily continuous. We use the machinery, based on the Scott extension formula, developed in [5, Long version, Appendix], and which we recall briefly now. By Claim Q of op.cit., for any stably compact space $Y$ (the result is stated for the slightly more general class of compact, stably core compact spaces), we may define a continuous functional $\mathfrak{r}(F)$ from $\left\langle Y \rightarrow \mathbb{R}^{+}\right\rangle$to $\overline{\mathbb{R}^{+}}$from any functional $F$ from $\left\langle Y \rightarrow \mathbb{R}^{+}\right\rangle$to $\overline{\mathbb{R}^{+}}$by the formula $\mathfrak{r}(F)(f)=\sup _{g \in B, g \ll f} F(g)$, where $B$ is a basis of the continuous poset $\left\langle Y \rightarrow \mathbb{R}^{+}\right\rangle$(described in Claim $\mathrm{K}$ ) and $\ll$ is its way-below relation; then $\mathfrak{r}(F)$ is the largest continuous functional below $F$, and is a continuous normalized linear prevision whenever $F$ is a normalized linear prevision.

In our case, observe that $Y=\mathbf{P}_{1 w k}^{\triangle}(X)$ is stably compact: the isomorphism $\alpha_{\mathcal{e}}$, $\gamma_{\mathcal{e}}$ between $\mathbf{P}_{1}^{\triangle}(X)$ and $\mathbf{V}_{1}(X)$ also defines an isomorphism between $\mathbf{P}_{1 w k}^{\triangle}(X)$ and $\mathbf{V}_{1 w k}(X)$ (where the latter is defined with the weak topology, whose subbasic opens are $[f>r]=\left\{p \in \mathbf{V}_{1}(X) \mid \oint_{x \in X} f(x) d p>r\right\}$ are in one to one correspondence to those of $\left.\mathbf{P}_{1 w k}^{\triangle}(X)\right)$. And $\mathbf{V}_{1 w k}(X)$ is stably compact as soon as $X$ is, a result 
due to Jung [9, Theorem 3.2]. So the machinery applies: $\mathcal{G}=\mathfrak{r}\left(\mathcal{G}_{0}\right)$ is a continuous normalized linear prevision, and $\mathcal{G} \leq \mathcal{G}_{0}$. Moreover, since $\mathcal{G}$ is the largest continuous functional below $\mathcal{G}_{0}$, and $q$ is continuous, we have $q \leq \mathcal{G} \leq \mathcal{G}_{0} \leq p$.

Using the isomorphism $\alpha_{\mathfrak{e}}, \gamma_{\mathfrak{e}}$, let $\mathcal{P}=\gamma_{\mathfrak{e}}(\mathcal{G})$ : this is a continuous normalized valuation on $\mathbf{P}_{1 w k}^{\triangle}(X)$. We claim it is supported on 2 . For every open $\mathcal{U}$ containing $\mathcal{Q}$ indeed, $q\left(\chi_{u}\right) \leq \mathcal{G}\left(\chi_{u}\right)$. But $q\left(\chi_{u}\right)=\min _{G^{\prime} \in \mathcal{Q}} \chi_{u}\left(G^{\prime}\right)=1$, and $\mathcal{G}\left(\chi_{u}\right)=\mathcal{P}(\mathcal{U})$. Since $\mathcal{P}(\mathcal{U}) \leq 1$ anyway, $\mathcal{P}(\mathcal{U})=1$.

For every $f \in\left\langle X \rightarrow \mathbb{R}^{+}\right\rangle$, let $\varphi$ be the function mapping $G^{\prime}$ to $G^{\prime}(f)$. Note that $\mathcal{G}(\varphi)=\alpha_{\mathfrak{e}}(\mathcal{P})(\varphi)=\oint_{G^{\prime} \in \mathbf{P}_{1 w k}^{\triangle}(X)} \varphi\left(G^{\prime}\right) d \mathcal{P}=\oint_{G^{\prime} \in \mathbf{P}_{1 w k}^{\triangle}(X)} G^{\prime}(f) d \mathcal{P}=\operatorname{Bary}(\mathcal{P})(f)$,

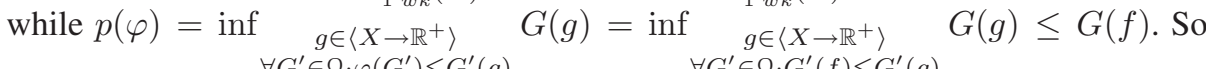
$\operatorname{Bary}(\mathcal{P}) \leq G$, as announced.

Let $\operatorname{Conv}(\mathcal{Q})$, the strong convex closure of $\mathcal{Q}$, be $\{\operatorname{Bary}(\mathcal{P}) \mid \mathcal{P}$ supported on $\mathcal{Q}\}$. Using Proposition 2 we may characterize the action of $\operatorname{CCoeur}_{1} \circ \Pi$ by $\operatorname{CCoeur}_{1}(\Pi \mathcal{Q})=$ $\uparrow \operatorname{Conv}(\mathcal{Q})$ for every $\mathcal{Q} \in \mathcal{Q}\left(\mathbf{P}_{1 w k}^{\triangle}(X)\right)$. Recall that $\mathcal{Q} \subseteq \operatorname{CCoeur}_{1}(\Pi \mathcal{Q})$. By Proposition 11, it follows that $\operatorname{Conv}(\mathcal{Q}) \subseteq C_{\text {Coeur }} \leq 1(\Pi \mathcal{Q})$. Since the heart is upward-closed, $\uparrow \operatorname{Conv}(\mathcal{Q}) \subseteq \operatorname{CCoeur}_{1}\left(\operatorname{CQ}^{2}\right)$. Conversely, if $G \in \operatorname{CCoeur}_{1}(\Pi \mathcal{Q})$, i.e., $\Pi \mathcal{Q} \leq G$, then by Proposition 2 there is a continuous probability valuation $\mathcal{P}$ supported on 2 , such that $\operatorname{Bary}(\mathcal{P}) \leq G$. This means that $\operatorname{Bary}(\mathcal{P}) \in \operatorname{Conv}(\mathcal{Q})$, so $G \in \uparrow \operatorname{Conv}(\mathcal{Q})$.

Theorem 1 (Isomorphism). Let $X$ be stably compact. Then CCoeur C $_{1}$ and $\prod$ define an isomorphism between $\nabla \mathbf{P}_{1}(X)$ and the space $Q^{C v x}\left(\mathbf{P}_{1 \text { wk }}^{\triangle}(X)\right)$ of strongly convex non-empty compact saturated subsets of $\mathbf{P}_{1 \text { wk }}^{\triangle}(X)$, ordered by $\supseteq$.

Proof. It is enough to realize that for every $\mathcal{Q} \in \mathcal{Q}^{C v x}\left(\mathbf{P}_{1 w k}^{\triangle}(X)\right), \uparrow \operatorname{Conv}(\mathcal{Q})=$ $\uparrow \mathcal{Q}=\mathcal{Q}$, while CCoeur $_{1}(\Pi \mathcal{Q})=\uparrow \operatorname{Conv}(\mathcal{Q})$. The identity $\prod \circ$ CCoeur $_{1}=$ id is already known from [5].

\section{The Cpo Case}

We may refine Theorem 1 and replace strong convexity by the mere notion of convexity, when $X$ is a coherent, continuous and pointed (hence stably compact) cpo. This comes close to the results of Keimel and Plotkin [10], who show that the space of superadditive, positively homogeneous and Scott-continuous functionals from $\left[X \rightarrow \overline{\mathbb{R}^{+}}\right]$to $\overline{\mathbb{R}^{+}}$, is isomorphic to $Q^{c v x}(\overline{\mathbf{V}}(X))$, where $[X \rightarrow Y]$ denotes the cpo of all continuous maps from $X$ to $Y$ (not just the bounded ones), and $\overline{\mathbf{V}}(X)$ is the set of all valuations (not just the normalized ones, not even those that are bounded, i.e. do not take the value $+\infty$ ). Note also the use of $Q^{c v x}$ here instead of $Q^{C v x}$. Despite the apparent added generality of the results of [10], they do not seem to entail ours. (Try it!)

The key to our result is to realize that any compact saturated, convex subset of $\mathbf{P}_{1 w k}^{\triangle}(X)$ is in fact strongly convex. We need the following easily proved variant of [17. Theorem 3.8] first [6, Appendix A]. Call continuous cone any ordered cone $C$ which is continuous qua poset, and where + and $\cdot$ are Scott-continuous. (This is as the continuous d-cones of [17], except we don't require $C$ to be a cpo.) It is additive iff 
$x_{1} \ll y_{1}$ and $x_{2} \ll y_{2}$ imply $x_{1}+x_{2} \ll y_{1}+y_{2}$. Call a subset $Z$ of an additive continuous cone regular whenever $Z$ is continuous as a sub-partial order of $C$, and whenever $x, y \in Z$ are such that $x \ll_{Z} y$, then $x \ll_{C} y$, where $\ll_{Z}$ and $\ll_{C}$ are the way-below relations of $Z$ and $C$ respectively.

Proposition 3. Let $C$ be an additive continuous cone, and $Z$ a regular subspace of $C$. For every convex compact subset $K$ of $Z$, for every non-empty convex closed subset $F$ of $Z$ disjoint from $K$, there is $a \in \overline{\mathbb{R}^{+}}, a>1$, and a continuous linear function $f: C \rightarrow \overline{\mathbb{R}^{+}}$such that $f(z)>$ a for all $z \in K$ and $f(y) \leq 1$ for every $y \in F$.

Caveat: The topology of $Z$ is that induced by that of $C$ (whose opens are of the form $V \cap Z, V$ open in $C$ ), but there is no reason in general that this should coincide with the Scott topology of the ordering $\leq$ on $Z$. Such pathologies do not arise when $Z$ is a regular subspace of $C$ [6, Claim D, Appendix A]. For every continuous cpo $Y$, the space $C=\mathbf{V}(Y)$ of all continuous bounded valuations is an additive continuous cone, and $Z=\mathbf{V}_{\leq 1}(Y)$ is a regular subspace of $C$ [6, Claim F, Appendix A]. However, $\mathbf{V}_{1}(Y)$ is not regular in $\mathbf{V}(Y)$, when $Y$ has a least element $\perp$, as $\delta_{\perp} \ll \mathbf{V}_{1}(X) \delta_{\perp}$ but $\delta_{\perp} K_{\mathbf{V}(X)} \delta_{\perp}$ (the family $r \delta_{\perp}, r<1$, has the right-hand side as sup, but no element of this family is greater than or equal the left-hand side).

Recall that a saturated subset is one that is the intersection of all opens containing it. Say that a subset $A$ is linearly saturated iff $A$ is the intersection of all convex opens containing it. It is tempting to think that any convex saturated subset should be linearly saturated. This is indeed the case for those subsets that are also compact, as the following variant of [17, Corollary 3.13], due to Jung, shows.

Proposition 4. Let $C$ be a continuous cone, $Z$ be a regular convex subspace of $C$. Every convex compact saturated subset $Q$ of $Z$ is linearly saturated.

Proof. We must show that for every $x \in Z \backslash Q$, there is a convex open subset $V$ containing $Q$ but not $x$. Let $F=\{z \in Z \mid z \leq x\}$ : this is convex, non-empty, and disjoint from $Q$. Build $f$ and $a$ as in Proposition 3 . The open $V=f^{-1}(a,+\infty) \cap Z$ of $Z$ fits the bill. In particular, $V$ is convex because $f$ is linear.

Theorem 2. Let $X$ be a continuous pointed cpo. Every convex compact saturated subset $\mathcal{Q}$ of $\mathbf{P}_{1}^{\triangle}(X)=\mathbf{P}_{1 w k}^{\triangle}(X)$ is strongly convex.

Proof. Fix $X$, with least element $\perp$. By Edalat's variant of Jones' Theorem [2, Section 3], $Y=\mathbf{V}_{1}(X)$ is then also a continuous pointed cpo, with least element $\delta_{\perp}$. Using a trick by Edalat, $X^{\prime}=X \backslash\{\perp\}$ is a continuous cpo again, and $\mathbf{V}_{1}(X)$ is isomorphic to $\mathbf{V}_{\leq 1}\left(X^{\prime}\right)$. (Send $\nu \in \mathbf{V}_{1}(X)$ to $\lambda U \in \mathcal{O}\left(X^{\prime}\right) \cdot \nu(U)$, and send back $\nu \in \mathbf{V}_{\leq 1}\left(X^{\prime}\right)$ to $\nu^{\prime}$ defined as $\nu^{\prime}(U)=\nu(U)$ if $U \neq X$, i.e., $\perp \notin U, \nu^{\prime}(U)=1$ otherwise.) Take $C=\mathbf{V}\left(X^{\prime}\right), Z=\mathbf{V}_{\leq 1}\left(X^{\prime}\right)$. Let $\mathcal{Q}$ be a convex saturated compact of $Z, \mathcal{P}$ an element of $\mathbf{V}_{1}(Z)$ supported on 2 . Since $Z$ is a continuous pointed cpo again, we use again one of Edalat's results [2, Section 3]: every element of $\mathbf{V}_{1}(Z)$ is the sup of a directed family of simple probability valuations $\mathcal{P}_{i}, i \in I$. Fix an arbitrary convex open $\mathcal{U}$ containing 2. By definition of the Scott topology, some $\mathcal{P}_{i}$ is in $\mathcal{U}$, from which we deduce easily that the subfamily of those $\mathcal{P}_{i}$ that are in $\mathcal{U}$ is again directed, with sup $\mathcal{P}$. Let $J$ be the set of indices $i$ such that $\mathcal{P}_{i} \in \mathcal{U}$. However, since $\mathcal{U}$ is convex and $\mathcal{P}_{i}$ is simple, the finite 
barycenter $\operatorname{Bary}\left(\mathcal{P}_{i}\right)$ is in $\mathcal{U}$. It is easy to see that the family $\left(\operatorname{Bary}\left(\mathcal{P}_{i}\right)\right)_{i \in J}$ is directed. Its sup is in $\mathcal{U}$, since $\mathcal{U}$ is upward-closed. Since Choquet integration is continuous in the valuation argument, Bary is continuous, so this sup is just $\operatorname{Bary}(\mathcal{P})$. We have shown that $\operatorname{Bary}(\mathcal{P}) \in \mathcal{U}$ for every convex open $\mathcal{U}$ containing $\mathcal{Q}$. By Proposition $4, \mathcal{Q}$ is the intersection of all such convex opens, so $\operatorname{Bary}(\mathcal{P}) \in \mathcal{Q}$. This shows that every convex compact saturated subset of $Z=\mathbf{V}_{\leq 1}\left(X^{\prime}\right)$ is strongly convex. Now note that $Z$ is isomorphic to $\mathbf{V}_{1}(X)$, which is isomorphic to $\mathbf{P}_{1}^{\triangle}(X)=\mathbf{P}_{1 w k}^{\triangle}(X)$.

Since convex and strong convexity coincide for compact saturated subsets, the following is immediate.

Corollary 1 (Isomorphism). Let $X$ be a continuous, coherent pointed cpo. CCoeur $_{1}$ and $\Pi$ define an isomorphism between $\nabla \mathbf{P}_{1}(X)$ and $Q^{c v x}\left(\mathbf{P}_{1 \text { wk }}^{\triangle}(X)\right)=$ $Q^{c v x}\left(\mathbf{P}_{1}^{\triangle}(X)\right) \cong Q^{c v x}\left(\mathbf{V}_{1}(X)\right)$.

Note that $C$ Coeur 1 and $\Pi$ also exhibit $\nabla \mathbf{P}_{1}(X)$ as a retract of $\mathcal{Q}\left(\mathbf{P}_{1}^{\triangle}(X)\right)$, i.e., they are continuous, and $\Pi \circ C_{\text {Coeur }}=\mathrm{id}$. ( $\Pi$ is the retraction, and CCoeur C $_{1}$ the associated section.) By [2], $\mathbf{P}_{1}^{\triangle}(X) \cong \mathbf{V}_{1}(X)$ has a basis of simple normalized linear previsions, i.e., previsions of the form $\alpha_{\mathrm{e}}(p)$, with $p$ a simple probability valuation. Concretely, these are previsions of the form $\lambda h \in\left\langle X \rightarrow \mathbb{R}^{+}\right\rangle \cdot \sum_{i=0}^{n} a_{i} h\left(x_{i}\right)$, where $a_{0}, a_{1}, \ldots, a_{n} \in \mathbb{R}^{+}, \sum_{i=0}^{n} a_{i}=1$. It is well-known that, whenever $Y$ is a continuous cpo, $\mathcal{Q}(Y)$ is also a continuous cpo with basis given by the finitary compacts $\uparrow \mathcal{E}, \mathcal{E}$ a finite subset of $Y$ [1]. It is also known that any retract $Z^{\prime}$ of a continuous cpo $Z$ is again a continuous cpo, with basis given by the image of any basis of $Z$ by the retraction. So:

Theorem 3. For any continuous, coherent pointed cpo $X, \nabla \mathbf{P}_{1}(X)$ is a continuous, coherent pointed cpo. A basis is given by previsions of the form $\lambda h \in\left\langle X \rightarrow \mathbb{R}^{+}\right\rangle$. $\min _{i=1}^{m} \sum_{j=1}^{n} a_{i j} h\left(x_{j}\right)$, where $a_{i j} \in \mathbb{R}^{+}$and $\sum_{j=1}^{n} a_{i j}=1$ for each $i$.

Proof. The only things that remain to be proved are that $\nabla \mathbf{P}_{1}(X)$ has a least element $\left(\lambda h \cdot h(\perp)\right.$, i.e., $\left.\alpha_{\mathcal{C}}\left(\delta_{\perp}\right)\right)$, and that $\nabla \mathbf{P}_{1}(X)$ is coherent. Note that $\mathcal{Q}\left(\mathbf{P}_{1}^{\triangle}(X)\right)$ is a bc-domain, i.e., a continuous cpo where any two elements $Q_{1}$ and $Q_{2}$ having an upper bound have a least upper bound (namely $Q_{1} \cap Q_{2}$, which is non-empty because $Q_{1}$ and $Q_{2}$ have an upper bound $Q \subseteq Q_{1}, Q_{2}$ ). Every bc-domain is coherent, hence stably compact (see, e.g., [8]), and by a result of Lawson quoted by Jung [9], every retract of a stably compact space is again stably compact.

The above theorem means that we can always approximate, from below, any continuous normalized lower prevision by one that is computable, using only finitely many min, + and - operations. It is remarkable that we know no proof of this fact that would avoid the relatively daunting constructions above.

\section{Angelic Non-determinism + Probabilistic Choice}

Let the Hoare powerdomain $\mathcal{H}(Y)$ be the set of all non-empty closed subsets of $Y$, ordered by inclusion (a standard model of angelic non-determinism alone). This is also a cpo, which is continuous as soon as $Y$ is, and is usually used to model angelic 
non-determinism. Let $\triangle \mathbf{P}_{1}(X)$ be the space of all continuous normalized upper previsions on $X$. Then [5, Proposition 5] there is a map $C P e a u_{1}: \triangle \mathbf{P}_{1}(X) \rightarrow \mathcal{H}\left(\mathbf{P}_{1 \text { wk }}^{\triangle}(X)\right)$, defined by $C \operatorname{Peau}_{1}(F)=\left\{G \in \mathbf{P}_{1}^{\triangle}(X) \mid G \leq F\right\}$, and a map $\bigsqcup: \mathcal{H}\left(\mathbf{P}_{1 w k}^{\triangle}(X)\right) \rightarrow$ $\triangle \mathbf{P}_{1}(X)$, sending $\mathcal{F}$ to $\lambda h \in\left\langle X \rightarrow \mathbb{R}^{+}\right\rangle \cdot \sup _{G \in \mathcal{F}} G(h)$. When $X$ is stably compact, $\bigsqcup \dashv C P e a u_{1}$ defines what we called a Galois surrection, i.e., $\bigsqcup$ and $C P e a u_{1}$ are monotonic, $\bigsqcup \circ C P e a u_{1}=\mathrm{id}$, and $C P e a u_{1} \circ \bigsqcup(\mathcal{F}) \supseteq \mathcal{F}$ for all $\mathcal{F} . \bigsqcup$ is continuous, but we do not know whether $C P e a u_{1}$ is continuous in general.

We shall prove that $\bigsqcup$ and $C P e a u_{1}$ define an isomorphism similar to those of the previous sections. The main trick is in using a nice duality between demonic and angelic non-determinism, which we called convex-concave duality on games in [4], and which extends to previsions. Very roughly, the idea is to turn any prevision $F$ into the functional $F^{\perp}=\lambda h \in\left\langle X \rightarrow \mathbb{R}^{+}\right\rangle \cdot-F(-h)$. If $F$ is lower, then $F^{\perp}$ will be upper, and conversely, moreover $F^{\perp \perp}=F$. Unfortunately, $F(-h)$ is in general ill-defined: First, $-h$ does not take its values in $\mathbb{R}^{+}$(easy to repair, see below); second, $-h$ is very far from being continuous from $X$ to $\mathbb{R}^{+}$: the inverse image of the (Scott-)open $(t,+\infty)$ by $-h$ is $h^{-1}(-\infty,-t)$, of which we know nothing.

To correct the first problem, extend any normalized prevision $F$ on $X$ to a functional $\widehat{F}:\langle X \rightarrow \mathbb{R}\rangle \rightarrow \mathbb{R}$ by letting $\widehat{F}(f)=F(f+a)-a$, for any $a \geq-\inf _{x \in X} f(x)$. (As before, $\langle X \rightarrow \mathbb{R}\rangle$ is the space of all bounded continuous maps from $X$ to $\mathbb{R}$, with the Scott topology of the pointwise ordering.) This is independent of $a$, because $F$ is normalized. It is easy to see that $\widehat{F}$ is monotonic (if $f \leq f^{\prime}$ then $\widehat{F}(f) \leq \widehat{F}\left(f^{\prime}\right)$ ), positively homogeneous (if $r \geq 0$ then $\widehat{F}(r f)=r \widehat{F}(f)$ ), normalized, and lower, resp. upper, resp. linear, resp. Scott-continuous when $F$ is.

Solving the second problem is harder. We will have to approximate functions $-h$ with $h \in\left\langle X \rightarrow \mathbb{R}^{+}\right\rangle$by functions $g$ not from $X$, but from the de Groot dual $X^{\mathrm{d}}$ of $X$, to $\mathbb{R}^{+}$. This is defined (when $X$ is stably compact) as $X$, only with the socalled cocompact topology, whose opens are the cocompacts, i.e., subsets of the form $X \backslash Q, Q$ compact saturated subset of $X$. Observe that well-filternedness, coherence, and compactness imply that this is indeed a topology. Then $X^{\mathrm{d}}$ is again stably compact, and $X^{\mathrm{dd}}=X$ (see [9] for more background material on this).

A function $f: X \rightarrow \mathbb{R}$ is a step function if and only if it is of the form $\sum_{i=0}^{n} a_{i} \chi_{U_{i}}$, where $X=U_{0} \supseteq U_{1} \supseteq \ldots \supseteq U_{n}$ is a sequence of opens, and $a_{0} \in \mathbb{R}, a_{1}, \ldots, a_{n} \in$ $\mathbb{R}^{+}$. It is well-known (see e.g., [15]) that any element $f$ of $\langle X \rightarrow \mathbb{R}\rangle$ is the sup of a directed family of step functions, namely $f_{K}=a+\frac{1}{2^{K}} \sum_{k=1}^{\left\lfloor(b-a) 2^{K}\right\rfloor} \chi_{f-1}\left(a+\frac{k}{2^{K}},+\infty\right)$, $K \in \mathbb{N}$, where $a$ is any lower bound for $f$ and $b$ is any upper bound for $f$.

Definition $1\left(F^{\perp}\right)$. Let $X$ be a stably compact space, $F$ a normalized prevision on $X$. The dual $F^{\perp}$ of $F$ is the map from $\left\langle X^{\mathrm{d}} \rightarrow \mathbb{R}^{+}\right\rangle$to $\mathbb{R}^{+}$defined by $F^{\perp}(g)=$ $-\inf _{f \text { step function }} \widehat{F}(f)$.

We sum up the main properties of this construction. The proof is omitted for lack of space, but can be found in [6, Appendix B].

Theorem 4. Let $X$ be a stably compact space. For every normalized prevision $F$ on $X, F^{\perp}$ is a normalized prevision on $X^{\mathrm{d}}$. Moreover: (1) $F^{\perp}$ is continuous; (2) if $F$ is 
lower, then $F^{\perp}$ is upper; (3) if $F$ is upper, then $F^{\perp}$ is lower; (4) if $F$ is linear, then so is $F^{\perp}$; (5) if $F$ is continuous, then $F^{\perp \perp}=F$; (6) if $F \leq F^{\prime}$ then $F^{\perp} \leq F^{\perp}$.

The main step is to show that, when $g$ is a step function, $F^{\perp}(g)$ can be defined alternatively as $-\inf _{f} \sqsupseteq^{\mathrm{d}-g} \widehat{F}(f)$, where $\sqsupseteq^{\mathrm{d}}$ is a more constrained relation: $f \sqsupseteq^{\mathrm{d}}-g$ iff one can write $f$ as $-\sum_{i=0}^{n} a_{i} \chi_{X \backslash U_{i}}\left(U_{i}\right.$ opens, $\emptyset=U_{0} \subseteq U_{1} \subseteq \ldots \subseteq U_{n}, a_{0} \in \mathbb{R}$, $\left.a_{1}, \ldots, a_{n} \in \mathbb{R}^{+}\right), g$ as $\sum_{i=0}^{n} a_{i} \chi_{X \backslash Q_{i}}\left(Q_{i}\right.$ compact saturated subsets, $\emptyset=Q_{0} \subseteq$ $Q_{1} \subseteq \ldots \subseteq Q_{n}$ ), with the same coefficients $a_{i}$, and $Q_{i} \subseteq U_{i}$ for each $i$. While the proofs require $\sqsupseteq^{d}$, we observe that Definition 1 can be simplified by eliminating the recourse to step functions [6, Appendix C].

Lemma 1. Let $X$ be a stably compact space, and $F$ a normalized continuous prevision on $X$. For every $g \in\left\langle X^{\mathrm{d}} \rightarrow \mathbb{R}\right\rangle, F^{\perp}(g)=-\inf _{f \in\langle X \rightarrow \mathbb{R}\rangle} \widehat{F}(f)$.

$$
f \geq-g
$$

For every continuous game $\nu$ in the sense of [4] (in particular, a continuous valuation) on a stably compact space, we may define $\nu^{\perp}$ as $\gamma_{\mathcal{e}}\left(F^{\perp}\right)$ where $F=\alpha_{\mathcal{C}}(\nu)$. One may check that $\nu^{\perp}(X \backslash Q)=1-\nu^{\dagger}(Q)$, where $\nu^{\dagger}(Q)=\inf _{U \supseteq Q} \nu(U)$ [6, Claim X, Appendix B]. In the case where $\nu$ is a continuous valuation, the $\nu^{\dagger}$ construction was already studied by Tix [15, Satz 3.4].

Proposition 5. Let $X$ be stably compact. The map $p \mapsto p^{\perp}$ defines an isomorphism between $\mathbf{V}_{1 w k}(X)^{\mathrm{d}}$ and $\mathbf{V}_{1 w k}\left(X^{\mathrm{d}}\right)$. The map $F \mapsto F^{\perp}$ defines an isomorphism between $\mathbf{P}_{1 w k}^{\triangle}(X)^{\mathrm{d}}$ and $\mathbf{P}_{1 w k}^{\triangle}\left(X^{\mathrm{d}}\right)$.

Proof. The second claim follows from the first through the isomorphism $\alpha_{e}, \gamma_{e}$. For any compact saturated subset $Q$ of $X$, for any real $r$, let $\langle Q<r\rangle=\left\{p \in \mathbf{V}_{1}(X) \mid p^{\dagger}(Q)\right.$ $<r\}$. By [9, Concluding remarks], the sets $\langle Q<r\rangle$ form a subbasis of the cocompact topology on $\mathbf{V}_{1 w k}(X)$, provided $X$ is stably compact. (This is stated in terms of sets written $[K \geq r]$, which are the complements of $\langle K<r\rangle$. See [7, Section 6.4] for a proof.) So $p \mapsto p^{\perp}$ is continuous. Then apply Theorem 4 (6).

For lower previsions, we used probability valuations $\mathcal{P}$ supported on a compact saturated subsets $\mathcal{Q}$ of $\mathbf{P}_{1 w k}^{\triangle}(X)$. Upper previsions require us to use cosupports instead. Say that $\mathcal{P}$ is co-supported on $\mathcal{F} \subseteq \mathbf{P}_{1 w k}^{\triangle}(X)$ iff $\mathcal{P}(\mathcal{U})=0$, where $\mathcal{U}$ is the complement of the closure $\operatorname{cl}(\mathcal{F})$ of $\mathcal{F}$. It is easy to see that $\mathcal{P}$ is supported on the compact saturated subset $\mathcal{Q}$ of $\mathbf{P}_{1 w k}^{\triangle}(X)$ iff $\mathcal{P} \perp$ is co-supported on the closed subset $\mathcal{Q}$ of $\mathbf{P}_{1 w k}^{\triangle}(X)^{\mathrm{d}}$.

Say that a subset $\mathcal{F}$ of $\mathbf{P}_{1 w k}^{\triangle}(X)$ is co-strongly convex iff $\operatorname{Bary}(\mathcal{P}) \in \mathcal{F}$ for every $\mathcal{P} \in \mathbf{V}_{1}\left(\mathbf{P}_{1 w k}^{\triangle}(X)\right)$ that is co-supported on $\mathcal{F}$. When $\mathcal{P}$ is simple, say $\mathcal{P}=\sum_{i=0}^{n} a_{i} \delta_{G_{i}}$, then $\mathcal{P}$ is co-supported on $\mathcal{F}$ (when $\mathcal{F}$ is downward-closed) iff every $G_{i}$ such that $a_{i} \neq$ 0 is in $\mathcal{F}$; so every co-strongly convex downward-closed set is convex. We shall use this notion when $\mathcal{F}$ is a closed subset of $\mathbf{P}_{1 w k}^{\triangle}(X)$, in which case $\mathcal{F}$ will always be downward-closed. By an argument similar to that of Proposition 1 [6, Appendix D]:

Proposition 6. Let $X$ be stably compact, and $F \in \triangle \mathbf{P}_{1}(X)$. Then $C \operatorname{Peau}_{1}(F)$ is co-strongly convex.

The key argument for the converse is the following proposition, which states how Bary behaves w.r.t. the dualizing operation _ ${ }_{-}^{\perp}$. For any continuous map $f: Y \rightarrow Z$, and 
every $p \in \mathbf{V}_{1 w k}(Y)$, the image, a.k.a. push-forward continuous valuation $f[p] \in$ $\mathbf{V}_{1 w k}(Z)$ is that which sends each $V \in \mathcal{O}(Z)$ to $p\left(f^{-1}(V)\right)$. The change-of-variables formula for Choquet integration states that $\oint_{z \in Z} g(z) d f[p]=\oint_{y \in Y} g(f(y)) d p$ (an easy consequence of the definition). We use the notation ${ }_{-}^{\perp}\left[\mathcal{P}^{\prime}\right]$ below (with $\mathcal{P}^{\prime}=\mathcal{P}^{\perp}$ ), where__ $: \mathbf{P}_{1 w k}^{\triangle}(X)^{\mathrm{d}} \rightarrow \mathbf{P}_{1 w k}^{\triangle}\left(X^{\mathrm{d}}\right)$.

Proposition 7. Let $X$ be stably compact. For any continuous probability valuation $\mathcal{P}$ on $\mathbf{P}_{1 w k}^{\triangle}(X),\left(\operatorname{Bary}\left({ }_{-}{ }^{\perp}\left[\mathcal{P}^{\perp}\right]\right)\right)^{\perp}=\operatorname{Bary}(\mathcal{P})$.

Proof. Using the change-of-variables formula, Bary $\left({ }_{-}^{\perp}\left[\mathcal{P}^{\perp}\right]\right)=\lambda g \in\left\langle X^{\mathrm{d}} \rightarrow \mathbb{R}^{+}\right\rangle$. $\oint_{G^{\prime} \in \mathbf{P}_{1 w k}^{\triangle}\left(X^{\mathrm{d}}\right)} G^{\prime}(g) d_{-}^{\perp}[\mathcal{P} \perp]=\lambda g \in\left\langle X^{\mathrm{d}} \rightarrow \mathbb{R}^{+}\right\rangle \cdot \oint_{G \in \mathbf{P}_{1 w k}^{\triangle}(X)^{\mathrm{d}}} G^{\perp}(g) d \mathcal{P}^{\perp}=$ $\lambda g \in\left\langle X^{\mathrm{d}} \rightarrow \mathbb{R}^{+}\right\rangle \cdot-\inf _{\varphi \in\left\langle\mathbf{P}_{1 w k}^{\triangle}(X) \rightarrow \mathbb{R}\right\rangle} \oint_{G \in \mathbf{P}_{1 w k}^{\triangle}(X)} \varphi(G) d \mathcal{P}$ (using the definition $\varphi \geq \lambda G \in \mathbf{P}_{1 w k}^{\triangle}(X)^{\mathrm{d}} \cdot-G^{\perp}(g)$

of $\mathcal{P}^{\perp}$ as $\alpha_{\mathcal{C}}\left(\mathcal{P}^{\perp}\right)=\alpha_{\mathcal{C}}(\mathcal{P})^{\perp}$, and Lemma1).

For each $g \in\left\langle X^{\mathrm{d}} \rightarrow \mathbb{R}\right\rangle$, and each constant $a \geq-\inf _{x \in X} g(x)$, then, Bary( $\left.\left.\widehat{{ }^{\perp}[\mathcal{P} \perp}\right]\right)$

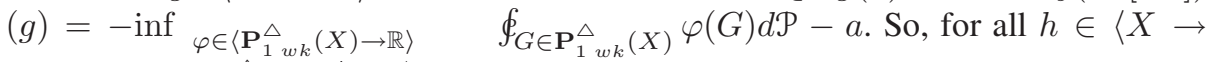
$\varphi \geq \lambda G \in \mathbf{P}_{1 w k}^{\triangle}(X)^{\mathrm{d}} \cdot-G^{\perp}(g+a)$

$\left.\mathbb{R}^{+}\right\rangle$, with $a \geq \sup _{x \in X} h(x)$, we obtain:

$$
\left(\operatorname{Bary}\left(-^{\perp}\left[\mathcal{P}^{\perp}\right]\right)\right)^{\perp}(h) \sup _{\substack{g \in\left\langle X^{\mathrm{d}} \rightarrow \mathbb{R}\right\rangle \\ g \geq-h}} \inf _{\substack{\varphi \in\left\langle\mathbf{P}_{1}^{\triangle}{ }_{w k}(X) \rightarrow \mathbb{R}\right\rangle \\ \varphi \in \mathbf{P}_{1 w k}^{\triangle}(X)^{\mathrm{d}} \cdot-G^{\perp}(g+a)}} \oint_{G \in \mathbf{P}_{1 w k}^{\triangle}(X)} \varphi(G) d \mathcal{P}+a
$$

using Lemma 1. For every open $U$ in $X$, we claim that $\left(\operatorname{Bary}\left({ }_{-}^{\perp}\left[\mathcal{P}^{\perp}\right]\right)\right)^{\perp}\left(\chi_{U}\right) \leq$ $\operatorname{Bary}(\mathcal{P})\left(\chi_{U}\right)$. For every $g \in\left\langle X^{\mathrm{d}} \rightarrow \mathbb{R}\right\rangle$ with $g \geq-\chi_{U}$, we have $\chi_{U} \geq-g$, so (since $G^{\perp}$ is normalized) $G^{\perp}(g+a)=G^{\perp}(g)+a=\sup _{f \geq-g}-G(f)+a \geq$ $-G\left(\chi_{U}\right)+a$. For every $\varphi \in\left\langle\mathbf{P}_{1 w k}^{\triangle}(X) \rightarrow \mathbb{R}\right\rangle$ with $\varphi \geq \lambda G \in \mathbf{P}_{1 w k}^{\triangle}(X)^{\mathrm{d}} \cdot G\left(\chi_{U}\right)-a$,

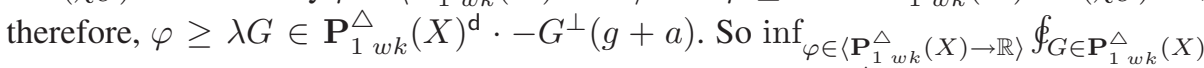
$\varphi \geq \lambda G \in \mathbf{P}_{1 w k}^{\Delta}(X)^{\mathrm{d}} \cdot G\left(\chi_{U}\right)-a$

$\varphi(G) d \mathcal{P} \geq \inf _{\varphi \in\left\langle\mathbf{P}_{1 w k}^{\triangle}(X) \rightarrow \mathbb{R}\right\rangle} \oint_{G \in \mathbf{P}_{1 w k}^{\triangle}(X)} \varphi(G) d \mathcal{P}$. The left-hand side is exactly $\varphi \geq \lambda G \in \mathbf{P}_{1 w k}^{\triangle}(X)^{\mathrm{d}} \cdot-G^{\perp}(g+a)$

$\operatorname{Bary}(\mathcal{P})\left(\chi_{U}\right)-a$ : take $\varphi=\lambda G \in \mathbf{P}_{1 w k}^{\triangle}(X)^{\mathrm{d}} \cdot G\left(\chi_{U}\right)-a$, and use the fact that $\operatorname{Bary}(\mathcal{P})$ is normalized. (The key point is that this $\varphi$ is indeed in $\left\langle\mathbf{P}_{1 w k}^{\triangle}(X) \rightarrow \mathbb{R}\right\rangle$, in particular continuous, as the inverse image of $(t,+\infty)$ is the open $\left[\chi_{U}>a+t\right]$.) So, using (1), $\left(\operatorname{Bary}\left({ }_{-}^{\perp}\left[\mathcal{P}^{\perp}\right]\right)\right)^{\perp}\left(\chi_{U}\right) \leq \sup _{g \in\left\langle X^{\mathrm{d}} \rightarrow \mathbb{R}\right\rangle} \operatorname{Bary}(\mathcal{P})\left(\chi_{U}\right)-a+a=$ $\operatorname{Bary}(\mathcal{P})\left(\chi_{U}\right)$.

Conversely, let us show that, for any two opens $U$ and $V$ of $X$ with $V \Subset U$, $\operatorname{Bary}(\mathcal{P})\left(\chi_{V}\right) \leq\left(\operatorname{Bary}\left({ }_{-}^{\perp}\left[\mathcal{P}^{\perp}\right]\right)\right)^{\perp}\left(\chi_{U}\right)$. The relation $\Subset$ is the way-below relation on $\mathcal{O}(X)$, ordered by inclusion; on every locally compact space, $U \Subset V$ iff $U \subseteq Q \subseteq V$ for some compact saturated set $Q$, and $\mathcal{O}(X)$ is then a continuous cpo [3]. Let $Q$ be a compact saturated subset such that $V \subseteq Q \subseteq U$. Then $g=-\chi_{Q}$ satisfies $g \geq-\chi_{U}$, so $\left(\operatorname{Bary}\left({ }_{-}^{\perp}\left[\mathcal{P}^{\perp}\right]\right)\right)^{\perp}\left(\chi_{U}\right) \geq \inf \quad \varphi \in\left\langle\mathbf{P}_{1 w k}^{\triangle}(X) \rightarrow \mathbb{R}\right\rangle \quad \oint_{G \in \mathbf{P}_{1 w k}^{\triangle}(X)} \varphi(G) d \mathcal{P}+a$. $\varphi \geq \lambda G \in \mathbf{P}_{1 w k}^{\triangle}(X)^{\mathrm{d}} \cdot-G^{\perp}\left(a-\chi_{Q}\right)$ 
Let us estimate $-G^{\perp}\left(a-\chi_{Q}\right)=\inf _{f \in\left\langle X \rightarrow \mathbb{R}^{+}\right\rangle} G(f)$ (using Lemma1). Whenever $f \geq$ $f \geq-\left(a-\chi_{Q}\right)$

$-\left(a-\chi_{Q}\right)$, we have $f \geq \chi_{Q}-a \geq \chi_{V}-a$, so $-G^{\perp}\left(a-\chi_{Q}\right) \geq \inf _{f \in\left\langle X \rightarrow \mathbb{R}^{+}\right\rangle} G(f) \geq$ $f \geq \chi_{V}-a$

$G\left(\chi_{V}-a\right)=G\left(\chi_{V}\right)-a$, since $G$ is normalized. Using (1), $\left(\operatorname{Bary}\left({ }_{-}{ }^{\perp}\left[\mathcal{P}^{\perp}\right]\right)\right)^{\perp}\left(\chi_{U}\right) \geq$ $\inf _{\varphi \in\left\langle\mathbf{P}_{1 w k}^{\triangle}(X) \rightarrow \mathbb{R}\right\rangle} \oint_{G \in \mathbf{P}_{1 w k}^{\triangle}(X)} \varphi(G) d \mathcal{P}+a=\oint_{G \in \mathbf{P}_{1 w k}^{\triangle}(X)} G\left(\chi_{V}\right) d \mathcal{P}-a+a=$ $\varphi \geq \lambda G \in \mathbf{P}_{1 w k}^{\Delta}(X)^{\mathrm{d}} \cdot G\left(\chi_{V}\right)-a$

$\operatorname{Bary}(\mathcal{P})\left(\chi_{V}\right)$.

Let $p=\gamma_{\mathcal{C}}(\operatorname{Bary}(\mathcal{P})), p^{\prime}=\gamma_{\mathcal{e}}\left(\left(\operatorname{Bary}\left({ }_{-}{ }^{\perp}\left[\mathcal{P}^{\perp}\right]\right)\right)^{\perp}\right)$. We have shown that $p^{\prime}(U) \leq$ $p(U)$ for every open $U$ of $X$, and that $p^{\prime}(U) \geq p(V)$ whenever $V \Subset U$. Since $\mathcal{O}(X)$ is a continuous cpo, and $p$ is continuous, $p(U)=\sup _{V \Subset U} p(V) \leq p^{\prime}(U)$. So $p=p^{\prime}$. Since $\alpha_{\mathcal{e}}, \gamma_{\mathcal{e}}$ form an isomorphism, $\operatorname{Bary}(\mathcal{P})=\left(\operatorname{Bary}\left({ }_{-}^{\perp}\left[\mathcal{P}^{\perp}\right]\right)\right)^{\perp}$.

Let $\operatorname{Conv}^{*}(\mathcal{F})$, the co-strong convex closure of $\mathcal{F}$, be $\{\operatorname{Bary}(\mathcal{P}) \mid \mathcal{P}$ co-supported on $\mathcal{F}\}$, and $\downarrow$ be the downward-closure operator. Similarly to Section 3 we show that $\operatorname{CPeau}_{1}(\bigsqcup \mathcal{F})=\downarrow \operatorname{Conv}^{*}(\mathcal{F})$ for every $\mathcal{F} \in \mathcal{H}\left(\mathbf{P}_{1 w k}^{\triangle}(X)\right)$. It is easy to see that (on stably compact $X$ ), for any normalized continuous upper prevision $F$ on $X$, $C_{P_{e a u}}(F)^{\perp}=C_{\text {Coeur }}\left(F^{\perp}\right)$; this is because $\_\perp$ is antitone (Theorem 4 (6)). For every non-empty closed subset $\mathcal{F}$ of $\mathbf{P}_{1 w k}^{\triangle}(X), C \operatorname{Peau}_{1}(\bigsqcup \mathcal{F})^{\perp}=\operatorname{CCoeur}_{1}\left((\bigsqcup \mathcal{F})^{\perp}\right)$ $=\operatorname{CCoeur}_{1}\left(\Pi \mathcal{F}^{\perp}\right)$ (because ${ }_{-}^{\perp}$ is antitone) $=\uparrow \operatorname{Conv}\left(\mathcal{F}^{\perp}\right)$ (see Section 3). Apply ${ }_{-}^{\perp}$, using Theorem 4 (5) to get $\operatorname{CPeau}(\bigsqcup \mathcal{F})=\uparrow \operatorname{Conv}\left(\mathcal{F}^{\perp}\right)^{\perp}=\downarrow \operatorname{Conv}\left(\mathcal{F}^{\perp}\right)^{\perp}=$ $\downarrow \operatorname{Conv}^{*}(\mathcal{F})$. The latter equality is a straightforward exercise, using Proposition 7 and the easily proved facts that, for any continuous function $f: Y \rightarrow Z$, for any continuous probability valuation $p$ on $Y$ : (a) if $p$ is supported on some subset $A$ of $X$, then $f[p]$ is supported on the direct image $f(A)$; (b) if $f[p]$ is co-supported on some closed subset $F$ of $Y$, then $p$ is co-supported on $f^{-1}(F)[6$, Appendix E].

Theorem 5 (Isomorphism). Let $X$ be stably compact. Then $C P e a u_{1}$ and $\bigsqcup$ define an isomorphism between $\triangle \mathbf{P}_{1}(X)$ and the space $\mathcal{H}^{C v x^{*}}\left(\mathbf{P}_{1 \text { wk }}^{\triangle}(X)\right)$ of co-strongly convex non-empty closed subsets of $\mathbf{P}_{1 \text { wk }}^{\triangle}(X)$, ordered by $\subseteq$.

The case where $X$ is a cpo is much simpler than for the demonic case (Section 4 ).

Lemma 2. Let $X$ be a continuous pointed cpo. Every convex closed subset of $\mathbf{P}_{1}^{\triangle}(X)$ is co-strongly convex.

Proof. Let $Z=\mathbf{P}_{1}^{\triangle}(X), \mathcal{F}$ a convex closed subset of $Z$, and $\mathcal{P}$ a continuous probability valuation on $Z$, co-supported on $\mathcal{F}: \mathcal{P}(Z \backslash \mathcal{F})=0$. Since $Z \cong \mathbf{V}_{1}(X)$ is a continuous pointed cpo, $\mathbf{V}_{1}(Z)$ is one, too, with a basis of simple probability valuations [2]. So write $\mathcal{P}$ as the sup of a directed family $\left(\mathcal{P}_{i}\right)_{i \in I}$, with $\mathcal{P}_{i} \leq \mathcal{P}$. In particular, $\mathcal{P}_{i}(Z \backslash \mathcal{F})=$ 0 , so $\mathcal{P}_{i}$ is co-supported on $\mathcal{F}$. Write $\mathcal{P}_{i}$ as $\sum_{j=1}^{n} a_{j} \delta_{G_{j}}$, where each $G_{j}$ is in $\mathcal{F}$, and $a_{1}+\ldots+a_{n}=1 . \mathcal{F}$ is convex so $\operatorname{Bary}\left(\mathcal{P}_{i}\right)=\sum_{j=1}^{n} a_{j} G_{j}$ is in $\mathcal{F}$. Now Bary is continuous, so $\operatorname{Bary}(\mathcal{P})=\sup _{i \in I} \operatorname{Bary}\left(\mathcal{P}_{i}\right)$. As $\mathcal{F}$ is $\operatorname{Scott}-\operatorname{closed}, \operatorname{Bary}(\mathcal{P}) \in \mathcal{F}$.

Writing $\mathcal{H}^{c v x}(Y)$ the subset of $\mathcal{H}(Y)$ consisting of convex subsets, it follows: 
Corollary 2 (Isomorphism). Let $X$ be a continuous, coherent pointed cpo. CPeau and $\sqcup$ define an isomorphism between $\triangle \mathbf{P}_{1}(X)$ and $\mathcal{H}^{c v x}\left(\mathbf{P}_{1 w k}^{\triangle}(X)\right)=$ $\mathcal{H}^{c v x}\left(\mathbf{P}_{1}^{\triangle}(X)\right) \cong \mathcal{H}^{c v x}\left(\mathbf{V}_{1}(X)\right)$.

One may also show the following [6, Appendix F]. This depends crucially on the fact that $C \operatorname{Peau}_{1}(\bigsqcup \mathcal{F})=\downarrow \operatorname{Conv}^{*}(\mathcal{F})$.

Proposition 8. Let $X$ be a continuous, coherent, pointed cpo. Then $C P e a u_{1}$ is a continuous map from $\triangle \mathbf{P}_{1}(X)$ to $\mathcal{H}\left(\mathbf{P}_{1 \text { wk }}^{\triangle}(X)\right)$.

Recall that, in this case, $Y=\mathbf{P}_{1 w k}^{\triangle}(X)=\mathbf{P}_{1}^{\triangle}(X)$ has a basis of simple normalized linear previsions. For any continuous cpo $Y, \mathcal{H}(Y)$ is continuous cpo too, with basis given by the finitary closed subsets $\downarrow \mathcal{E}, \mathcal{E}$ a finite subset of $Y$ [1]. As for Theorem 3 , we can therefore conclude:

Theorem 6. For any continuous, coherent pointed cpo $X, \triangle \mathbf{P}_{1}(X)$ is a continuous, coherent pointed cpo. A basis is given by previsions of the form $\lambda h \in\left\langle X \rightarrow \mathbb{R}^{+}\right\rangle$. $\max _{i=1}^{m} \sum_{j=1}^{n} a_{i j} h\left(x_{j}\right)$, where $a_{i j} \in \mathbb{R}^{+}$and $\sum_{j=1}^{n} a_{i j}=1$ for each $i$.

So we can also approximate from below any continuous normalized upper prevision by one that is computable, using only finitely $\max ,+$, and · operations.

\section{Chaotic Non-determinism + Probabilistic Choice}

In chaotic non-determinism we replace $\mathcal{Q}(Y)$ or $\mathcal{H}(Y)$ by the Plotkin powerdomain $\mathcal{P} \ell(Y)$. This is the set of all lenses $L$, which are non-empty intersections of a compact saturated subset $Q$ of $Y$ and a closed subset $F$ of $Y$. A canonical way of writing $L$ as $Q \cap F$ is then to take $Q=\uparrow L, F=c l(L)$. We order $\mathcal{P} \ell(Y)$ by the topological EgliMilner ordering $\Xi_{\mathrm{EM}}$ defined by $L \sqsubseteq \mathrm{EM} L^{\prime}$ iff $\uparrow L \supseteq \uparrow L^{\prime}$ and $\operatorname{cl}(L) \subseteq \operatorname{cl}\left(L^{\prime}\right)$. If $Y$ is a continuous, coherent pointed cpo, then $\mathcal{P} \ell(Y)$ is one, too. (See [1, Section 6.2.3].) Among all lenses, call strong those that obey the stronger property $F=\downarrow L$.

Let $\mathbf{F}_{1}(X)$ be the space of all normalized forks on $X$. Then [5, Proposition 6] there is a map $C C_{\text {Corps }}: \mathbf{F}_{1}(X) \rightarrow \mathcal{P} \ell\left(\mathbf{P}_{1 w k}^{\triangle}(X)\right)$, defined by $C \operatorname{Corps}_{1}\left(F^{-}, F^{+}\right)=$ $\operatorname{CCoeur}_{1}\left(F^{-}\right) \cap \operatorname{CPeau}_{1}\left(F^{+}\right)$. Moreover, $\operatorname{CCoeur}_{1}\left(F^{-}\right)=\uparrow \operatorname{CCorps}_{1}(F)$ and $C \operatorname{Peau}_{1}\left(F^{+}\right)=\downarrow \operatorname{CCorps}_{1}(F)$. (So CCorps ${ }_{1}\left(F^{-}, F^{+}\right)$is a strong lens.) It is clear

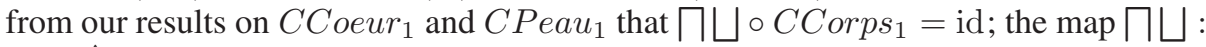
$\mathcal{P} \ell\left(\mathbf{P}_{1 w k}^{\triangle}(X)\right) \rightarrow \mathbf{F}_{1}(X)$ is defined by $\sqcap \bigsqcup \mathcal{L}=(\sqcap \mathcal{Q}, \sqcup \mathcal{C})$, where $\mathcal{Q}=\uparrow \mathcal{L}$ and $\mathcal{C}=$ $\operatorname{cl}(\mathcal{L})$. The following is an immediate consequence of the results of previous sections:

Proposition 9. A subset $\mathcal{A}$ of $\mathbf{P}_{1 \text { wk }}^{\triangle}(X)$ is bi-strongly convex iff for every continuous probability valuation $\mathcal{P}$ supported on $\mathcal{A}, \operatorname{Bary}(\mathcal{P})$ is in $\uparrow \mathcal{A}$, and for every continuous probability valuation $\mathcal{P}$ co-supported on $\mathcal{A}, \operatorname{Bary}(\mathcal{P})$ is in $\downarrow \mathcal{A}$.

Let $X$ be stably compact. For every lens $\mathcal{L}$ on $\mathbf{P}_{1 w k}^{\triangle}(X), C \operatorname{Corps}_{1}(\sqcap \bigsqcup \mathcal{L})=$ $\uparrow \operatorname{Conv}(\mathcal{L}) \cap \downarrow$ Conv $^{*}(\mathcal{L})$. CCorps ${ }_{1}$ and $\sqcap \bigsqcup$ define an isomorphism between $\mathbf{F}_{1}(X)$ and the space $\mathcal{P} L^{\text {biCvx }}\left(\mathbf{P}_{1 w k}^{\triangle}(X)\right)$ of all strong bi-strongly convex lenses on $\mathbf{P}_{1 w k}^{\triangle}(X)$. 
Let now $X$ be a continuous, coherent pointed cpo. It is an easy consequence of Theorem 2 and Lemma 2 that any strong lens $\mathcal{L}$ on $\mathbf{P}_{1 w k}^{\triangle}(X)$ is bi-strongly convex. Also, every lens is in fact strong [1, Lemma 6.2.20]. Write $\mathcal{P} \ell^{c v x}(Y)$ the subspace of convex lenses in $\mathcal{P} \ell(X)$ :

Theorem 7. Let $X$ be a continuous, coherent pointed cpo. CCorps $s_{1}$ and $\Pi \bigsqcup$ define an isomorphism between $\mathbf{F}_{1}(X)$ and $\mathcal{P} \ell^{c v x}\left(\mathbf{P}_{1 w k}^{\triangle}(X)\right)=\mathcal{P} \ell^{c v x}\left(\mathbf{P}_{1}^{\triangle}(X)\right) \cong$ $\mathcal{P} \ell^{c v x}\left(\mathbf{V}_{1}(X)\right)$.

\section{Conclusion}

We have solved the problem of relating domains of continuous previsions and forks à la [5], and convex powercones à la [17]: in standard cases, they are isomorphic. This question was raised under this form at the end of [5], while Keimel and Plotkin [10] show similar results for (unbounded) valuations instead of normalized valuations. They justify working on unbounded valuations because "the mathematics seems to be more natural if we take all the valuations, since one can then work with notions of linearity rather than convexity". I hope to have convinced the reader that the mathematics of the normalized case, once generalized to the topological case, is both beautiful and deep. Note in particular that the notion of (generalized) barycenters $\operatorname{Bary}(\mathcal{P})$, and above all the convex-concave duality work naturally at the topological level, not directly on cpos.

Acknowledgements. Thanks to the anonymous referees, and to Roberto Segala for their careful rereading.

\section{References}

1. Abramsky, S., Jung, A.: Domain theory. In: Abramsky, S., Gabbay, D.M., Maibaum, T.S.E. (eds.) Handbook of Logic in Computer Science, OUP, vol. 3, pp. 1-168 (1994)

2. Edalat, A.: Domain theory and integration. Theor. Comp. Sci. 151, 163-193 (1995)

3. Gierz, G., Hofmann, K.H., Keimel, K., Lawson, J.D., Mislove, M., Scott, D.S.: Continuous lattices and domains. In: Encycl. Mathematics and its Applications, CUP, vol. 93 (2003)

4. Goubault-Larrecq, J.: Continuous capacities on continuous state spaces. In: Arge, L., Cachin, C., Jurdziński, T., Tarlecki, A. (eds.) ICALP 2007. LNCS, vol. 4596, pp. 764-776. Springer, Heidelberg (2007)

5. Goubault-Larrecq, J.: Continuous previsions. In: Duparc, J., Henzinger, T.A. (eds.) CSL 2007. LNCS, vol. 4646, pp. 542-557. Springer, Heidelberg (2007)

6. Goubault-Larrecq, J.: Prevision domains and convex powercones. Research Report LSV-0733, Laboratoire Spécification et Vérification, ENS Cachan, France, 34 pages (October 2007)

7. Goubault-Larrecq, J.: Une introduction aux capacités, aux jeux et aux prévisions (June 2007), http://www.1sv.ens-cachan.fr/ goubault/ProNobis/pp_1_8.pdf

8. Jung, A.: Cartesian Closed Categories of Domains. PhD thesis, T.H. Darmstadt (1998)

9. Jung, A.: Stably compact spaces and the probabilistic powerspace construction. In: Desharnais, J., Panangaden, P. (eds.) CADE 1980. Electronic Notes in Theoretical Computer Science, vol. 87, Elsevier, Amsterdam (2004)

10. Keimel, K., Plotkin, G.: Predicate transformers for convex powerdomains. Mathematical Structures in Computer Science, pages 42 (submitted, 2007) 
11. Kirch, O.: Bereiche und Bewertungen. Diplom, T.H. Darmstadt (1993)

12. Mislove, M.: Topology, domain theory and theoretical computer science. Topology and Its Applications 89, 3-59 (1998)

13. Mislove, M.: Nondeterminism and probabilistic choice: Obeying the law. In: Palamidessi, C. (ed.) CONCUR 2000. LNCS, vol. 1877, pp. 350-364. Springer, Heidelberg (2000)

14. Roth, W.: Hahn-Banach type theorems for locally convex cones. J. Australian Math. Soc. 68(1), 104-125 (2000)

15. Tix, R.: Stetige Bewertungen auf topologischen Räumen. Diplom, T.H. Darmstadt (June 1995)

16. Tix, R.: Continuous D-Cones: Convexity and Powerdomain Constructions. PhD thesis, T.U. Darmstadt (1999)

17. Tix, R., Keimel, K., Plotkin, G.: Semantic domains for combining probability and nondeterminism. Electronic Notes in Theor. Comp. Sci. 129, 1-104 (2005) 